\title{
An Enhanced Particle Swarm Optimisation Method Integrated With Evolutionary Game Theory
}

\author{
Cédric Leboucher*, Hyo-Sang Shin ${ }^{\dagger}$, Rachid Chelouah ${ }^{\S}$, \\ Stéphane Le Ménec*, Patrick Siarry ${ }^{\ddagger}$, Mathias Formoso*, Antonios Tsourdos ${ }^{\dagger}$ and Alexandre Kotenkoff* \\ * MBDA France \\ 1, av. Réaumur, 92350 Le Plessis Robinson (France) \\ Email: \{cedric.leboucher ; stephane.le-menec ; mathias.formoso ; alexandre.kotenkoff\} \\ @ mbda-systems.com \\ ${ }^{\dagger}$ Cranfield University \\ College Rd, Cranfield, Bedford MK43 0AL, UK \\ Email: $\{$ h.shin ; a.tsourdos\} @ cranfield.ac.uk \\ ‡Université Paris-Est Créteil (UPEC), LISSI (EA 3956) \\ 61, av. du Général de Gaulle, 94010, Créteil (France) \\ Email: siarry@u-pec.fr \\ $\S$ Ecole Internationale des Sciences du Traitement de l'Information (EISTI) \\ av. du parc, 95000 Cergy (France) \\ Email: rachid.chelouah@eisti.eu
}

\begin{abstract}
This paper develops a novel particle swarm optimiser algorithm. The focus of this study is how to improve the performance of the classical particle swarm optimisation approach, i.e., how to enhance its convergence speed and capacity to solve complex problems while reducing the computational load. The proposed approach is based on an improvement of Particle Swarm Optimisation using Evolutionary Game Theory. This method maintains the capability of the particle swarm optimiser to diversify the particles' exploration in the solution space. Moreover, the proposed approach provides an important ability to the optimisation algorithm, that is adaptation of the search direction which improves the quality of the particles based on their experience. The proposed algorithm is tested on a representative set of continuous benchmark optimisation problems and compared with some other classical optimisation approaches. Based on the test results of each benchmark problem, its performance is analysed and discussed.
\end{abstract}

Index Terms-Optimisation methods, Operation Research, Evolutionary Game Theory, Particle Swarm Optimisation, Replicator dynamics

\section{INTRODUCTION}

Over the last few decades, numerous scientists have been inspired by the modelling of social interactions of animals to solve NP-hard optimisation problems. Although the communication among the different agents is limited to an exchange of basic information, it results in very effective team work. Particle Swarm Optimisation (PSO) is one of most well known and established approaches using this concept. The aim of the original PSO method proposed by Kennedy and Eberhart was to reproduce this social interaction among agents in order to solve non linear continuous optimisation problems [9] [10].

The PSO principle is based on sharing simple information such as fitness and position among neighbouring particles in order to compute velocity and position of a swarm of candidate solutions, named particles. The movement of the particles is based on a random linear combination of their own current velocity, and the relative position vectors of their own best position and the best known position of the neighbouring particles with respect to their current position. Random choice of the three weighting parameters of the linear combination keeps diversification of the particles' search.

The study in this paper was initiated from the idea that it could be possible to improve the convergence behaviour of the particles by properly selecting the three weighting parameters instead of randomly choosing them within the typical interval. Thanks to Evolutionary Game Theory (EGT), problem of choosing weighting parameters in the PSO framework has been converted into a mathematically well-defined problem. EGT has the property to converge to Nash equilibria considering 
mixed strategies, i.e. probability distributions on the PSO parameters. On this side, EGT allows to choose PSO parameters in a more optimal way. One of the main interests of using EGT for tuning PSO parameters is that EGT ensures stability thanks to the Maynard-Smith second property [14].

This idea was first introduced in our previous study [11], [12]. The approach of combining PSO with EGT is named EGPSO and the convergence properties of such approach were investigated in our previous study [13]. This study proved that EGPSO guarantees local convergence. Although the previous study showed improvement of convergence speed, the proposed method could suffer from premature convergence. The issue with the approach in our previous study is that the particles tend to be trapped in local optima as the dimension of the problem increases. The proposed approach in this paper, named C-EGPSO (Combined - Evolutionary Game Particle Swarm Optimisation), handles this issue by adapting the strategy of each particle instead of considering the same strategy for the entire swarm. Unlike the previous study in which all particles use the same method to compute weighting parameters, each particle in the updated approach evaluates its own weighting parameters and adapts the individual search directions. Thus, the diversity of the swarm is guaranteed by an adapted movement based on the particle's history to evaluate a given search direction and the interactions with the other particles in the swarm, as well as the intensification by optimising the search direction using EGT. As a consequence, it is expected that, in the first phase, the particles will explore and learn the solution space, and then this will make the proposed method more adaptive to optimisation problems and capable of providing better convergence. Moreover the number of calls of the cost function is sufficiently reduced compared to the required computational time to obtain the best strategy. Hence, the proposed approach in this study also significantly reduces the computational load compared with our previous EGPSO algorithm.

Note that the idea of C-ESPSO was first suggested in our previous study [13] after investigation of the convergence properties of EGPSO. However, the previous study provided only the rationale behind this proposition and did not examine its performance over typical benchmark test functions. As continuation of our previous work in [13], this paper refines how to determine the three weighting parameters from our previous work and performs extensive empirical tests based on the well-defined benchmark functions from the CEC-2015 conference [1] to investigate the performance of the proposed C-EGPSO algorithm.

The rest of the paper is organised as follows. The proposed method will be introduced in Section II along with a technical background for PSO and EGT. Section III details the improvement performed by C-EGPSO compared to SPSO 2011 and EGPSO. Then, the obtained results of the proposed algorithm on a set of benchmark functions will be analysed and compared to those from other selected algorithms found in literature in Section IV. Note that the set of benchmark functions are selected from the CEC-2015 conference [1]. Finally, conclusions of this study will be given in the last section.

\section{Design of C-EGPSO}

Since the authors are using SPSO 2011 proposed by M. Clerc in [7] to design the proposed algorithm, the principle of SPSO 2011 will be introduced in detail. The following subsection describes SPSO 2011 that M. Clerc designed in [7].

\section{A. SPSO's moving rules}

Let $X_{i}^{t}=\left[x_{i 1}^{t}, x_{i 2}^{t}, \ldots, x_{i D}^{t}\right]$, where $x_{i d}^{t} \in \mathbb{R}$ for $d=1, \ldots, D$, be a particle in a population of $N$ particles. The velocity of this particle is denoted as $V_{i}^{t}=$ $\left[v_{i 1}^{t}, v_{i 2}^{t}, \ldots, v_{i D}^{t}\right], v_{i d}^{t} \in \mathbb{R}$. Each particle communicates with its neighbourhood according to a communication network. This network (or topology) plays an important role in the convergence speed and the exploration. The analogy with Game Theory could be also made here since the swarm can be considered as a population of decision makers, where the frequency with which a particular decision made can change over time in response to the decisions made by all individuals in the population. The studies carried out in [15] and [5] describe in great detail the influence of the topology over the ability of the algorithm to explore a solution space.

From this topology, the best position $P_{g}^{t}=$ $\left[p_{g 1}^{t}, p_{g 2}^{t}, \ldots, p_{g D}^{t}\right]$, among the informants is defined. Each particle has a memory in which it saves the best explored solution by itself. The vector $P_{i}^{t}=$ $\left[p_{i 1}^{t}, p_{i 2}^{t}, \ldots, p_{i D}^{t}\right]$, denotes this position.

The state of one particle at the instant $t+1$ is obtained from the three previously described components: the current velocity $V_{i}^{t}$, its own memory $P_{i}^{t}$ and the best position $P_{g}^{t}$ among the informants of the particles.

Note that Monson and Seppi exposed in [16] the bias result from the dimension by dimension motion used in the original PSO algorithm. Indeed, it was shown that PSO performs better when the optimal region is located on one of the referential axis. Therefore, Clerc proposed to amend this motion equation in a geometric way to avoid the referential dependence [7]. This explains the introduction of the barycentre in the general motion equation of the particles.

Let $G$ denotes the iso-barycentre of the particles $X_{i}^{t}$, $X_{i}^{t}+c_{1} P_{i}^{t}$ and $X_{i}^{t}+c_{2} P_{g}^{t}$, where $c_{1}$ and $c_{2}$ denote two 
real coefficients. The coordinates of the barycentre $G$ can be obtained as:

$$
G=\frac{X_{i}^{t}+\left(X_{i}^{t}+c_{1}\left(P_{i}^{t}-X_{i}^{t}\right)+X_{i}^{t}+c_{2}\left(P_{g}^{t}-X_{i}^{t}\right)\right)}{3}
$$

Then, a point $X_{i}^{\prime}$ is randomly drawn in the hypersphere $H\left(G,\left\|G-X_{i}\right\|\right)$, which is centred on $G$ with a radius equal to $\left\|G-X_{i}\right\|$. It results in the velocity update equation:

$$
V_{i}^{t+1}=w V_{i}^{t}+X_{i}^{t}-X_{i}^{t}
$$

where $w$ denotes a real-valued coefficient representing the inertia of the particle and $X_{i}^{\prime}{ }^{t}$ represents the randomly drawn point in the hypersphere $H\left(G,\left\|G-X_{i}\right\|\right)$ at the instant $t$.

The position update equation is given by:

$$
X_{i}^{t+1}=w V_{i}^{t}+X_{i}^{\prime t}
$$

In the case where $P_{g}^{t}$ and $X_{i}^{t}$ are the same particle, the particle $P_{g}^{t}$ is simply ignored in the computation of $G$. Then $G$ becomes:

$$
G=\frac{X_{i}^{t}+\left(X_{i}^{t}+c_{1}\left(P_{i}^{t}-X_{i}^{t}\right)\right)}{2}
$$

Figure 1 shows the moving rules for the particle $X_{i}^{t}$.

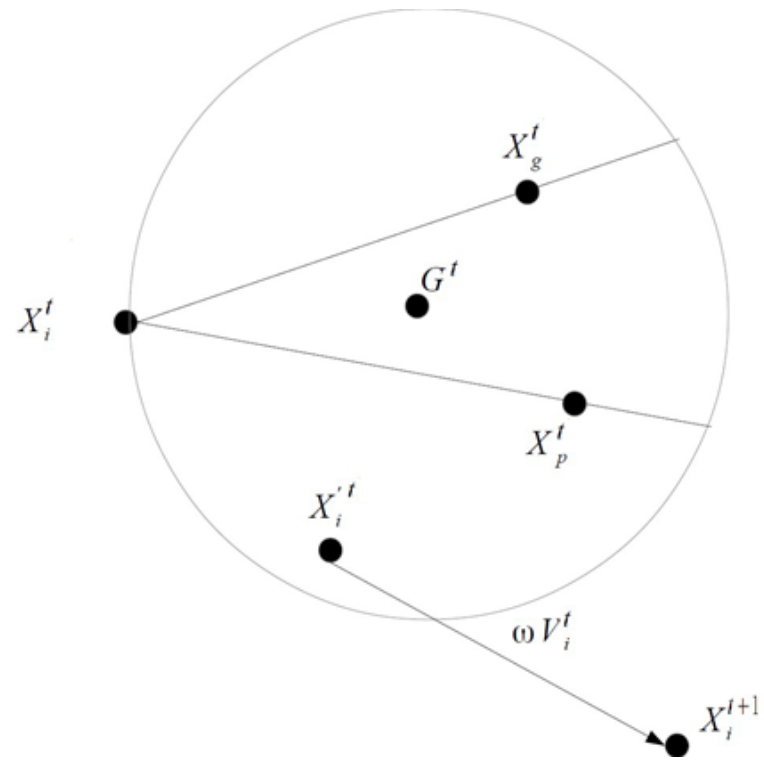

Fig. 1. Illustration of one possible particle move. $X_{i}$ represents the particle in its current state, $X_{i}$ represents its memory and $X_{g}$ denotes the best particle among the neighbourhood. $G$ is the iso-barycentre of the three previously enumerated particles. $X_{i}^{\prime}{ }^{t}$ is the randomly drawn point in the hypersphere $H\left(G,\left\|G-X_{i}\right\|\right)$. $w V_{i}^{t}$ denotes the inertia of the particle. $X_{i}^{t+1}$ represents the particle after the move.

In SPSO 2011 designed by M. Clerc [6], these coefficients are fixed to $w=\frac{1}{2 \log (2)}$ and $c_{1}=c_{2}=$ $0.5+\log (2)$. Note that these values were obtained by mathematical analysis on the stagnation in the PSO (no improvement in the best found solution). The proposed algorithm is using this work as starting point but uses a dynamical update of these coefficients to balance the intensification and the diversification of the search in the solution space. It will be detailed in the following subsection.

\section{B. Dynamical update of coefficients using Evolutionary Game}

1) Evolutionary stable strategies: In EGT, equilibrium is a key point to determine the output of a game. This equilibrium point is called the Evolutionary Stable Strategy (ESS).

An Evolutionary Stable Strategy is a strategy such that, if all members of a population adopt it, then no mutant strategy could invade the population under the influence of natural selection [8] [18]. Assume that we have a mixed population consisting of most of the agents playing optimal strategy $p^{*}$ with a few individuals using strategy $p$. That is, the strategy distribution in the population is:

$$
(1-\varepsilon) p^{*}+\varepsilon p
$$

where $\varepsilon>0$ is the small frequency of $p$ users in the population. Let the fitness $\pi$, i.e. payoff of an individual using strategy $q$ in this mixed population, be

$$
\pi\left(q,(1-\varepsilon) p^{*}+\varepsilon p\right) .
$$

Then, an interpretation of Maynard Smith's requirement [14] for $p^{*}$ to be an ESS is that, for all $p \neq p^{*}$,

$$
\pi\left(p,(1-\varepsilon) p^{*}+\varepsilon p\right)>\pi\left(p^{*},(1-\varepsilon) p^{*}+\varepsilon p\right)
$$

for all $\varepsilon>0$ "sufficiently small", for agents minimizing their fitness; note that the ESS always exists. However, it can be either a mixed strategy of the available strategies, or a pure strategy. A pure strategy means that the entire population will adopt it after a sufficient time.

Replicator dynamics

A common way to describe strategy interactions is using matrix games. Matrix games are described using notations as follows. $e_{i}$ is the $i^{t h}$ unit line vector of $i=1, \ldots, m$.

$A_{i j}=\pi\left(e_{i}, e_{j}\right)$ is the $m \times m$ payoff matrix.

$\Delta^{m} \equiv\left\{p=\left(p_{1}, \ldots, p_{m}\right) \mid p_{1}+\ldots+p_{m}=\right.$ $\left.1,0 \leq p_{i} \leq 1\right\}$ is the set of mixed strategies (probability distributions over the pure strategies $e_{i}$ ).

Then, $\pi(p, q)=p \cdot A q^{T}$ is the payoff of agents playing strategy $p$ facing agents playing strategy $q$.

Another interpretation is $\pi(p, q)$ being the fitness of a large population of agents playing pure strategies $(p$ describing the agent proportion in each behaviour inside a population) with respect to a large $q$ population.

The Replicator Equation (RE) is an Ordinary Differential Equation which was initially introduced by Taylor 
and Joncker in [20]. It expressed the difference between the fitness of a strategy and the average fitness in the population. Lower payoffs (agents are minimizers) bring faster reproduction in accordance with Darwinian natural selection process. The initial form was as follows:

$$
\dot{p}_{i}=-p_{i}\left(e_{i} \cdot A p^{T}-p \cdot A p^{T}\right)
$$

The RE for $i=1, \ldots, m$ describes the evolution of strategy frequencies $p_{i}$. Moreover, for every initial strategy distribution $p(0) \in \delta^{m}$, there is an unique solution $p(t) \in \delta^{m}$ for all $t \geq 0$ that satisfies the replicator equation. The replicator equation is the most widely used evolutionary dynamics. It was introduced for matrix games by Taylor and Jonker [20].

The reader is referred to [11] for more details about the replication process of the replicator, the setting of the payoff matrix and the convergence to an evolutionary stable strategy.

2) Implementation in PSO: EGT has been designed to explain common animal behaviours when they are competing for some food resources for example. The example of the Hawk and Dove game is among the most famous examples in EGT and helps understanding of the basis of the principle of individual adapting its strategy accordingly to the rewards that were previously obtained. Based on this principle, the analogy is made:

\begin{tabular}{cll}
\hline EGT & & Analogy in our method \\
\hline Population & $\longrightarrow$ & Swarm \\
Individual & $\longrightarrow$ & Particle \\
Strategies & $\longrightarrow$ & Follow the personal best par- \\
& & ticle, global best particle or \\
& & inertia \\
Payoff matrix $\longrightarrow$ & $\begin{array}{l}\text { Mean of the performance ob- } \\
\text { tained by following a specific } \\
\end{array}$ \\
& & strategy
\end{tabular}

Then, each particle is a player having three available strategies (following its memory, the best neighbour, or moving using only the inertia). The payoff matrix is defined by:

$$
\Pi=\left(\begin{array}{ccc}
\pi\left(s_{1}\right) & \frac{\pi\left(s_{1}\right)-\pi\left(s_{2}\right)}{2} & \frac{\pi\left(s_{1}\right)-\pi\left(s_{3}\right)}{2} \\
\frac{\pi\left(s_{2}\right)-\pi\left(s_{1}\right)}{2} & \pi\left(s_{2}\right) & \frac{\pi\left(s_{2}\right)-\pi\left(s_{3}\right)}{2} \\
\frac{\pi\left(s_{3}\right)-\pi\left(s_{1}\right)}{2} & \frac{\pi\left(s_{3}\right)-\pi\left(s_{2}\right)}{2} & \pi\left(s_{3}\right)
\end{array}\right)
$$

where $\pi\left(s_{i}\right), i \in\{1,2,3\}$ denotes the payoff that a particle can get by following only the strategy $i$. The payoff matrix $\Pi$ is built from the difference between the average obtained result by following one pure strategy.

The numerical value of $\pi\left(s_{i}\right)$ for each $i$ is obtained from the previous experience of the considered particle. For the following equations, we are focusing on only one particle of the swarm. Let $S^{t}=\left(Q_{i}^{t}\right), i \in\{1,2,3\}$ be the evolutionary stable strategy obtained for a given payoff matrix $\Pi$. The coefficients $Q_{i}^{t}, i \in\{1,2,3\}$, represent the ratio of strategy $i$ at time $t . f\left(X^{t}\right)$ denotes the fitness of one particle at the time $t$. Then, the payoff $\pi\left(s_{i}\right)$ for the strategy $i$ is proposed as:

$$
\pi\left(s_{i}\right)=\frac{1}{t} \sum_{k=1}^{t} Q_{i}^{k} \cdot f\left(X^{k}\right)
$$

where the first term $1 / t$ represents the mean value over the time. The second term, $\sum_{k=1}^{t} Q_{i}^{k} \cdot f\left(X^{k}\right)$ is the sum of the ESS strategies multiplied by the corresponding cost obtained for the particle $X$. The choice of these payoffs can be explained by the fact that it represents the mean value of the obtained gain by following a strategy. Since this strategy can be sometimes mixed, the $Q_{i}^{t}$ term enables the weight of the involvement of each strategy in the performance of the particle move.

Once the payoff matrix is filled, the Replicator Dynamics equation is applied to a fictitious population in order to obtain the associated ESS. This ESS is composed by the 3 ratios of each strategy. These ratios is then used to determine the three coefficients $w, c_{1}$ and $c_{2}$. The choice to use these coefficients in the proposed method can be explained by the fact that they represent a stable direction to follow. The stability feature of the ESS is used to address the possible irregularity of the solution space and make the exploration less susceptible to the influence of local optima. Since the particles are following a sort of instinct developed by their previous experiences, they will be able to "shape" the solution space to enhance the search direction.

To determine the convergence characteristics of the algorithm, the ESS is weighted by three coefficients. These coefficients were empirically tuned to improve the convergence speed. The mapping from the ESS to the three weighting parameters, $w, c_{1}$ and $c_{2}$, is determined as follows:

$$
\begin{gathered}
w=\left(\sigma_{M A X}-\sigma_{E S S}\right) \omega_{P S O} \\
c_{1}=3 Q_{2}^{t} \\
c_{2}=-3 Q_{2}^{t}+3
\end{gathered}
$$

where $Q_{i}^{t}(i \in\{1,2,3\})$ denotes the obtained ESS and $\omega_{P S O}$ is the inertia coefficient of the SPSO 2011 algorithm. Here, $\sigma_{M A X}$ denotes the maximum value for the standard deviation of mixed strategies that are bounded in $[0 ; 1]$ and $\sigma_{E S S}$ represents the standard deviation of the triplet $\left(Q_{1}^{t} ; Q_{2}^{t} ; Q_{3}^{t}\right)$. In the proposed framework, the maximum value of $\sigma_{M A X}$ is, for instance, the standard deviation of the set $[1,0,0]$ and is approximatively equal to 0.5774. Note that this mapping is the main difference of C-EGPSO from the original SPSO 2011 
[7] and our previous studies. Moreover, the proposed mapping is updated from our previous works [11]-[13] to further improve the convergence properties. The choice of this function enables to keep the balance among mixed strategies, and auto-eliminates those strategies with poor survival in the population game.

\section{The algorithm design}

The proposed method uses a fixed number of particles. This number should be carefully selected reflecting characteristics of the optimal problems. Moreover, this number should not be high to allow each particle to make a minimum number of moves within the number of function calls. For later comparison with SPSO 2011, the choice of this number comes from the study led by M. Clerc in [7].

The topology of the communication network is identical to that of the SPSO 2011. Each particle communicates with itself and has a probability $p_{\text {inf }}=1-(N-1 / N)^{K}$ to be an informant for the other particles. $K$ represents the maximum number of neighbours for one particle, so each particle is informed by at most $K$ other particles, with $K \in[0, N] \cap \mathbb{N}$.

To exploit the main benefit of EGPSO, i.e. the enhanced convergence speed, without suffering a premature convergence, we propose a random selection of the role that each particle follows. There are two possible roles: EGPSO described in subsection II-B and the classical SPSO 2011 described in subsection II-A. The role assignment is simply random, which implies that a particle has a probability $p_{R}$ to follow the EGPSO process, and $1-p_{R}$ to follow SPSO 2011. Therefore, we could keep the two key capabilities of PSO, i.e. exploration and exploitation capabilities. The EGPSO algorithm could help the intensification of the search from the improved convergence speed, whereas SPSO 2011 could help to diversify the search of the particles.

Figure 2 describes the principle of the proposed approach in the setting of optimal PSO coefficients. Note that EGPSO is obtained from C-EGPSO by setting the parameter $p_{R}$ to 1 .

The probability $p_{R}$ is empirically tuned by investigating the effect of $p_{R}$ on the optimization performance. Most of the CEC-2015 problems are tested for this investigation. This subsection shows the effect of $p_{R}$ on the optimization result over an example function, F5 of CEC-2015 problems. This example function is described in IV-A and its parameters for simulation are defined in IV-B. Different values of $p_{R}$, sampled through the interval $[0,1]$, were tested. The results are shown in Figure 3. From the investigation, it is concluded that the value of $1 / 3$ is a good compromise between fast convergence and exploration induced by game theory and SPSO.
Note that an automatic selection algorithm of $p_{R}$ should be a meaningful subject of the future study.

\section{C-EGPSO IMPROVEMENTS FROM SPSO}

\section{A. Influence of EGT on particle's exploration}

The herein subsection describes the role played by EGT in the exploration of the particles by analyzing the convergence of the ESS coefficients on different classes of problems.

Figure 4 is showing the convergence of particles toward the minimum of a 2D sphere problem. The departure point of the swarm is displayed in blue $(90,90)$. The swarm is composed of $\mathrm{N}=3$ particles and 20 iterations are displayed. Early iterations are plotted in green whereas latter ones are in red. Two phases can be seen clearly, a convergent phase where the swarm is descending to the minimum in 4 or 5 iterations following the gradient of the Sphere function. Then a latter phase where the particles, in red, are exploring the space around the minimum. Figure 5 shows quantitatively the evolution of the strategy ratio for the simple sphere problem. It can be seen that there are two stages in the choice of the strategies. In the earlier part of the simulation, the strategy that consists in following the best particle of the neighbourhood is dominant over the others that do not express at all. This can be explained by the global structure of the sphere problem. Indeed, in this simple unimodal problem, it is intuitive that sampling around the best solution will most likely improve the fitness of the particle. Then, in a second phase, when approaching the global minimum the memory as well as the inertia start appearing in the global strategy. This can be explained by the fact that, when the particles are close to the optimum, the swarm has converged and it improves the probability of sampling a point that is worst than the current position by following only the best particle. This demonstrates that the particles adapt themselves to the situation to always promote the strategy that will lead to the optimum solution.

Figure 6 shows the evolution of the strategy ratio when solving a Griewank's plus Rosenbrock's function which includes multiple local minima. In this example, we deliberately chose a failure case where the swarm is trapped in a local minimum. So, as for the previous example, there are two different phases in the strategy evolution. First, the particle uses very different strategies to quickly converge to a local minimum. Then, as soon as particles are in this local minimum, it can be seen that the strategies became balanced to stabilise around $[1 / 3,1 / 3,1 / 3]$. This equilibrium means that no strategy is prevalent over the others and it is logical to take into account that the particle is trapped in local minimum. Thus, whatever its next local move is, it will not improve the quality of the current solution. 


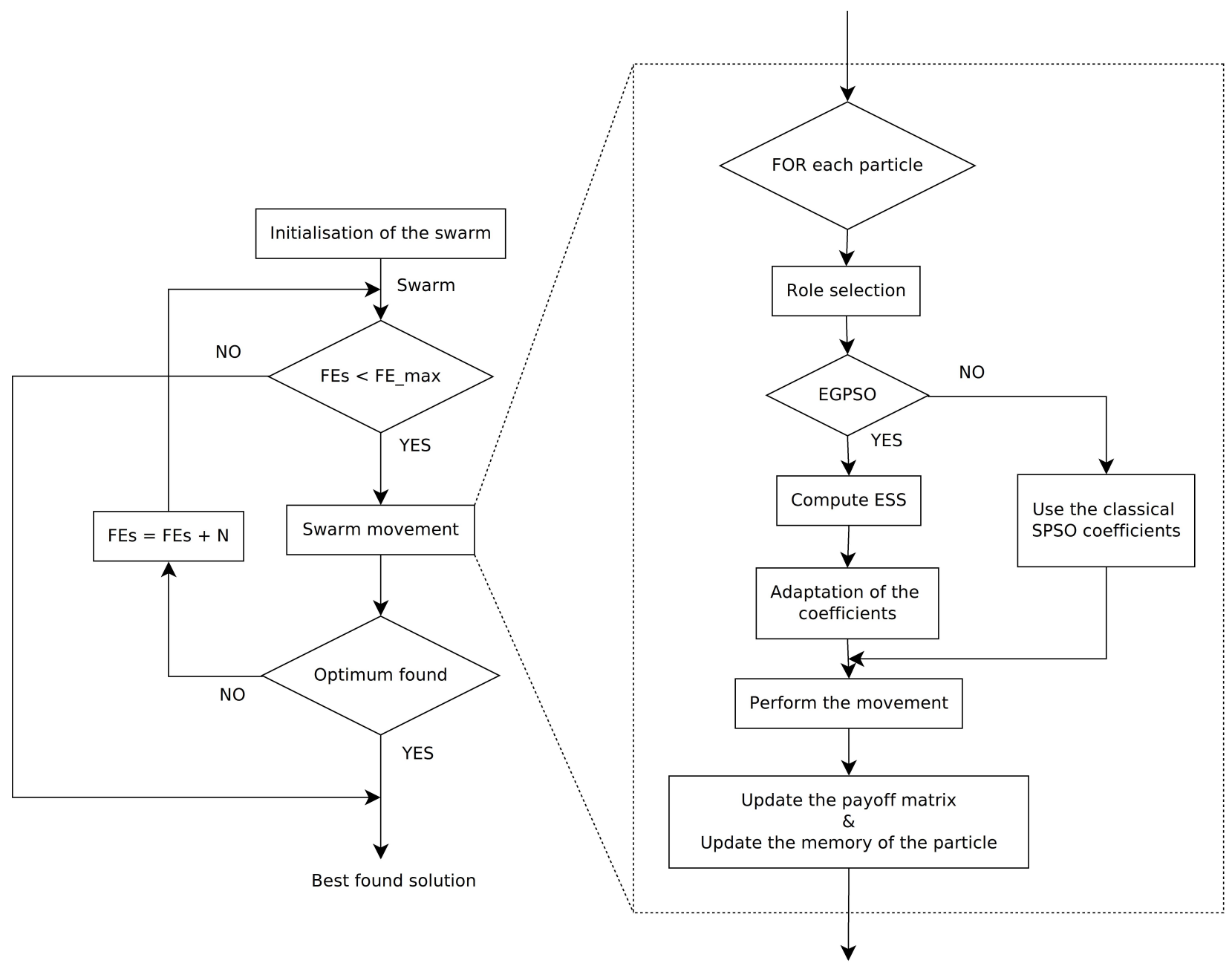

Fig. 2. Diagram of the proposed method. The values $F E S$ and $F E_{\max }$ denote the current number of cost function calls and the maximum of authorised calls respectively.

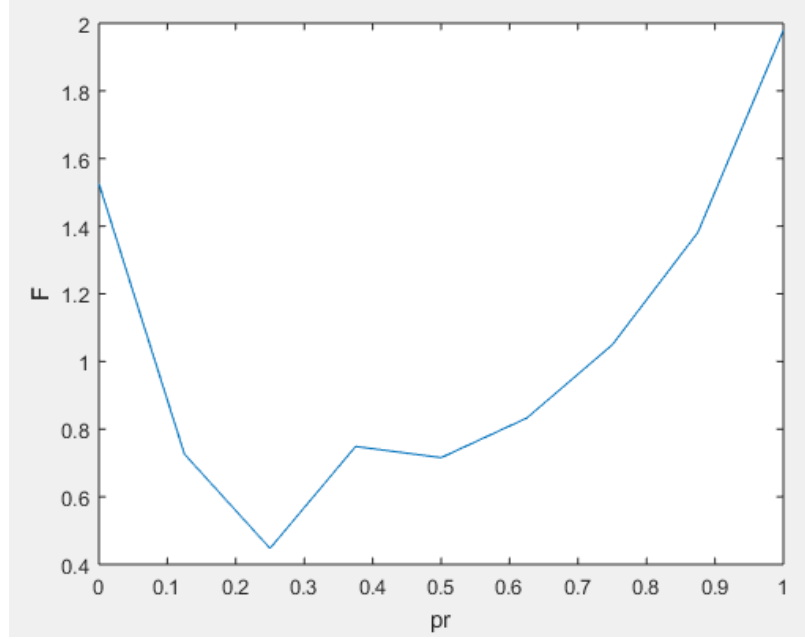

Fig. 3. Evolution of the mean, over 20 simulation runs, of the best results on problem F5 according to different values of $p_{R}$.
Hence, as illustrated by these two examples, it was shown that the adaptation of the particles to the situation was effective by using the EGT process. In the first case, it enables a fast convergence to the interesting area, then locally explores the area to find the global optimum. In the second example, it was demonstrated that the particles adopt a rational behaviour and if they are trapped in a local minimum, they restart a global exploration of the solution space by balancing the three classical components of the PSO.

\section{B. The local optima issue}

This subsection studies, on two problems, how the C-EGPSO overcomes a premature convergence issue encountered by the previous versions of EGPSO [11], [12]. Figure 7 is plotted for problem $F_{4}$ of the CEC2015 benchmark respectively for $D=10$ and $D=30$. $F_{4}$ features various local optima as well. It is a proper test to see how the adaptation of the three ESS coefficients 


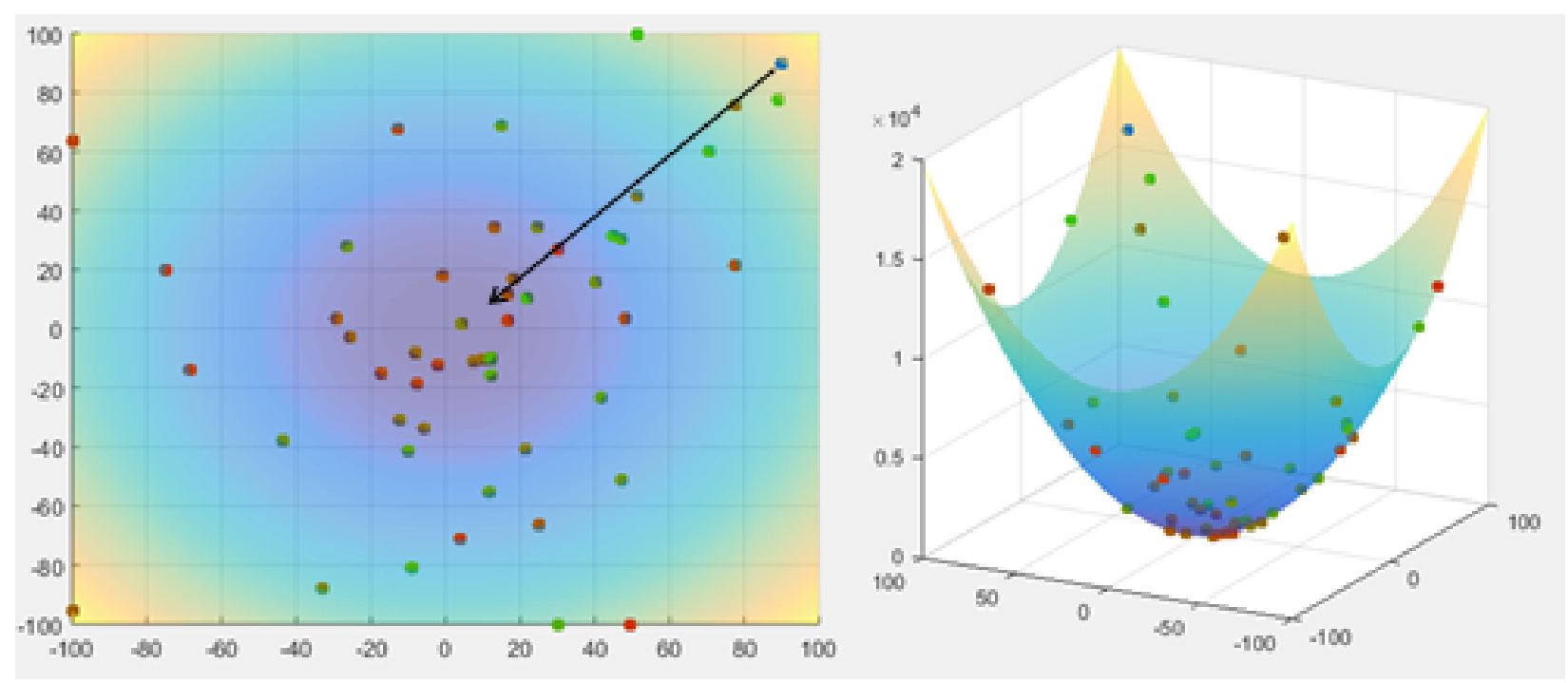

Fig. 4. Convergence of the C-EGPSO algorithm on a 2D Sphere Problem. Color of the particles are evolving from green to red according to the iteration number. The arrow indicates the convergence direction in early iterations.

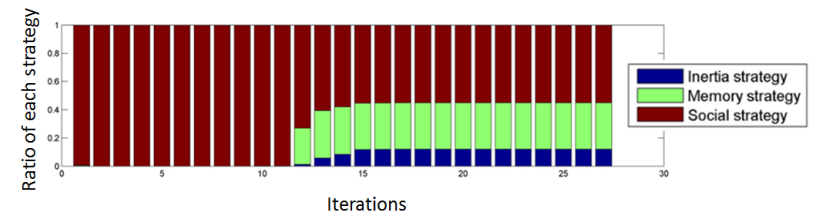

Fig. 5. Evolution of the strategies over the time for one randomly chosen particle. This example shows the ratio of each strategy over the time for a simple sphere problem.

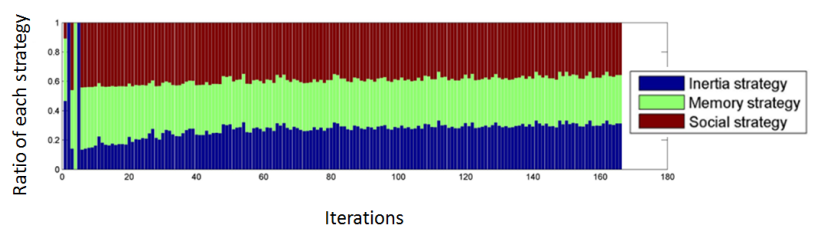

Fig. 6. Evolution of the strategies over the time for one randomly chosen particle. This example shows the ratio of each strategy over the time for a Griewank's plus Rosenbrock's function.

performs on complex problems. However, in order to have conclusive results, the population size was chosen as $N=40$ and the maximum number of function calls was set to $1000 D$. It shows, for the selected problems, the evolution of the results for different numbers of function evaluations (FES). The abscissa gives the percentage progression of FES.

The upper graphic of Figure 7 gives the convergence speeds of the compared methods for dimension $D=10$ on the problem $F_{4}$. SPSO 2011 performs a good exploration of the solution space with constant coefficients. However these coefficients were obtained
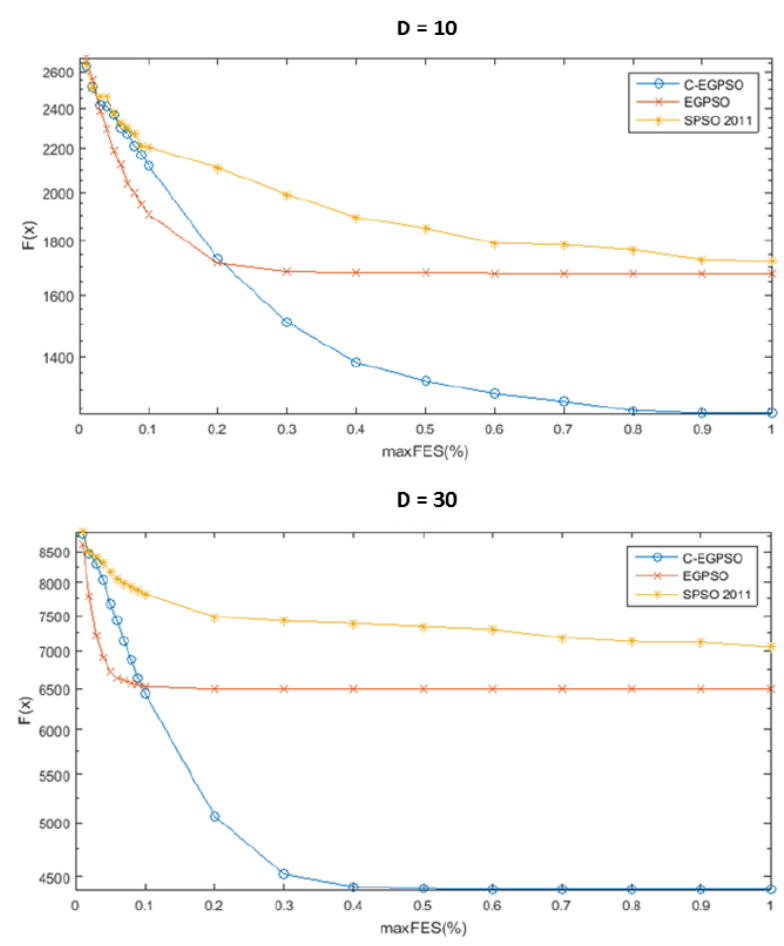

Fig. 7. Comparison of the convergence of the best value for problem $F_{4}$ of CEC2015 benchmark in dimension $D=10$ (top) and dimension $D=30$ (bottom).

by mathematical analysis on the stagnation in the PSO [6] and are not fitted with the solution space for early exploration. In this early exploration phase, C-EGPSO is driven by EGT and converges faster than SPSO 2011. 
It can be noticed that EGPSO is trapped after 2000 iterations. This can be explained by the fact that EGT proposes a locally optimal search direction. Thus, when all particles are trapped in the same local optima, it is more difficult for them to escape these areas. There is a lack of exploration in this case. However, the exploration properties of SPSO 2011 can be used to escape this local optimum. Hence, combination of both approaches using $p_{R}=1 / 3$ allows particles to have a good intensification while keeping the advantages of SPSO 2011 exploration property.

Taking the results of $D=30$ displayed on the bottom part of Figure 7, it is observed that C-EGPSO is less impacted by the dimension on problem $F_{4}$ than SPSO 2011. Actually, the dimension increases the complexity of the topology and thus enhances the ability of C-EGPSO to surpass local optima while keeping an adaptation of its coefficients to the topology of the problem.

To conclude this investigation, C-EGPSO improves SPSO 2011 on functions with numerous local optima especially on higher dimensions by using the mixed role of SPSO 2011 and EGPSO. It is also noticeable that the computational load remains almost unchanged since the experience of the particles is modelled by the storage of only 3 additional coefficients. This means that the designed approach keeps the simplicity of the interactions proposed in the initial PSO [9].

\section{BENCHMARK OF C-EGPSO}

\section{A. Definition of the CEC-2015 Benchmark}

In order to support the efficiency of the proposed method, the algorithm was tested on the benchmark functions issued from the CEC-2015 congress [1]. This includes two unimodal functions, seven multimodal functions, three hybrid functions and three composed functions. This heterogeneous set of problems can demonstrate the efficiency of the proposed approach to escape local minima and to converge to a global minimum in a reduced time. Due to the benchmark rules, the proposed approach is limited to 20 numerical simulations for each function and for dimensions $D=10$ and $D=30$. For each simulation, the number of cost function calls was limited to $50 D$, i.e. 500 for $D=10$ and 1500 for $D=30$. Then, the obtained results are compared to the other well-known competing algorithms results. The benchmark definition is available in [4]. In order to prevent algorithms from exploiting the symmetries of the solution space and to avoid the classical problem of the optimum value on the axis of the problem, the global optimum is shifted to a value different from zero and the function values of the global optima are non-zero. Moreover, all problems are rotated to test if the proposed method is using the coordinate system to perform the exploration of the solution space. In the proposed experiments, the tested functions are:

- $F_{1}$, Rotated Bent Cigar Function,

- $F_{2}$, Rotated Discus Function,

- $F_{3}$, Shifted and Rotated Weierstrass Function,

- $F_{4}$, Shifted and Rotated Schwefel Function,

- $F_{5}$, Shifted and Rotated Katsuura Function,

- $F_{6}$, Shifted and Rotated HappyCat Function,

- $F_{7}$, Shifted and Rotated HGBat Function,

- $F_{8}$, Shifted and Rotated Expanded Griewank's plus Rosenbrock's Function,

- $F_{9}$, Shifted and Rotated Expanded Schaffer F6 Function,

- $F_{10}$, Hybrid Function $1(\mathrm{~N}=3)$,

- $F_{11}$, Hybrid Function $2(\mathrm{~N}=4)$,

- $F_{12}$, Hybrid Function 3 (N=5),

- $F_{13}$, Composition Function $1(\mathrm{~N}=5)$,

- $F_{14}$, Composition Function $2(\mathrm{~N}=3)$,

- $F_{15}$, Composition Function $3(\mathrm{~N}=5)$.

N.B.: here, $\mathrm{N}$ denotes the number of functions used to obtain a hybrid or composed problem.

C-EGPSO will be compared to a Covariance Matrix Adaptation Evolution Strategy algorithm (CMAES-R) developed in [3], SPSO 2011 proposed by M. Clerc in [7], iSRPSO based on Self Regulated Particle Swarm Optimisation [19], HumanCog that is inspired by human cognitive and metacognitive behavior and defined in [2], and Mean-Variance Mapping Optimisation (MVMO) that constitutes an emerging heuristic optimisation algorithm, whose evolutionary mechanism adopts a single parentoffspring pair approach along with a normalised range of the search space for all optimisation variables [17]. The choice to compare our method with the aforementioned ones can be explained by the good results obtained by these algorithms on the proposed benchmark functions. Moreover, they represent different classes of algorithms and their performance provides an overview to the actual solutions of the problems of the benchmark.

For each problem, comparison will be made on the best result over the 20 numerical simulations.

\section{B. Experimental Setup}

For the simulation tests, the number of particles is set to $N=12$, the maximum number of neighbours each particle can have is set to be at most 3 neighbours, and the probability to be assigned to an EGPSO role is $p_{R}=\frac{1}{3}$. The selection of the coefficient $p_{R}$ is important to balance between the convergence speed and diversification of the search direction to avoid local optima while reducing exploration as it is described in subsection II-C. In this study, this coefficient was empirically tuned, i.e., we tried several values on several problems, then selected the one that obtained the 

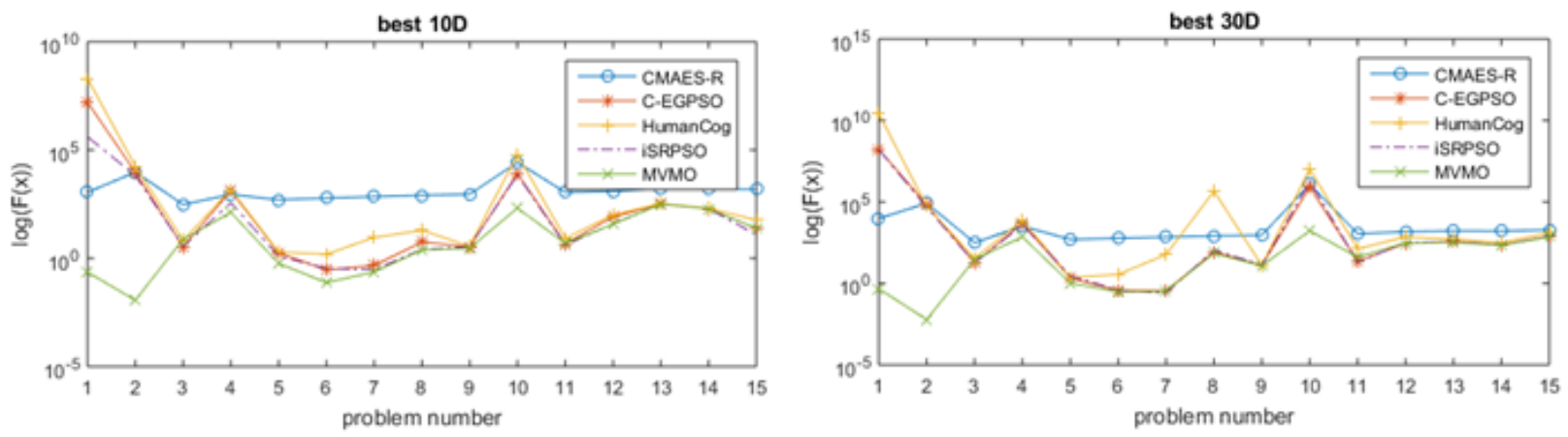

Fig. 8. Best values after 20 numerical simulations when running the CEC-2015 benchmark problems with parameter D equal to 10 and 30

\begin{tabular}{|c|c|c|c|c|c|c|c|}
\hline Func & Best & Worst & Median & Mean & Std & CMAES-R Best & CMAES-R Mean \\
\hline$F_{1}$ & $3.158 \mathrm{e}+06$ & $3.268 \mathrm{e}+07$ & $1.056 \mathrm{e}+07$ & $1.268 \mathrm{e}+07$ & $8.239 \mathrm{e}+06$ & $1.106 \mathrm{e}+03$ & $2.486 \mathrm{e}+06$ \\
$F_{2}$ & $7.155 \mathrm{e}+03$ & $6.802 \mathrm{e}+04$ & $3.200 \mathrm{e}+04$ & $3.418 \mathrm{e}+04$ & $1.392 \mathrm{e}+04$ & $9.478 \mathrm{e}+03$ & $3.725 \mathrm{e}+04$ \\
$F_{3}$ & $3.216 \mathrm{e}+00$ & $1.135 \mathrm{e}+01$ & $6.027 \mathrm{e}+00$ & $6.526 \mathrm{e}+00$ & $1.954 \mathrm{e}+00$ & $3.033 \mathrm{e}+02$ & $3.067 \mathrm{e}+02$ \\
$F_{4}$ & $9.851 \mathrm{e}+02$ & $2.083 \mathrm{e}+03$ & $1.755 \mathrm{e}+03$ & $1.691 \mathrm{e}+03$ & $3.095 \mathrm{e}+02$ & $8.827 \mathrm{e}+02$ & $1.912 \mathrm{e}+03$ \\
$F_{5}$ & $1.444 \mathrm{e}+00$ & $3.742 \mathrm{e}+00$ & $2.790 \mathrm{e}+00$ & $2.713 \mathrm{e}+00$ & $7.408 \mathrm{e}-01$ & $5.010 \mathrm{e}+02$ & $5.027 \mathrm{e}+02$ \\
$F_{6}$ & $2.002 \mathrm{e}-01$ & $9.430 \mathrm{e}-01$ & $5.482 \mathrm{e}-01$ & $5.689 \mathrm{e}-01$ & $1.484 \mathrm{e}-01$ & $6.003 \mathrm{e}+02$ & $6.005 \mathrm{e}+02$ \\
$F_{7}$ & $2.831 \mathrm{e}-01$ & $1.639 \mathrm{e}+00$ & $5.374 \mathrm{e}-01$ & $6.994 \mathrm{e}-01$ & $3.706 \mathrm{e}-01$ & $7.002 \mathrm{e}+02$ & $7.009 \mathrm{e}+02$ \\
$F_{8}$ & $2.675 \mathrm{e}+00$ & $7.560 \mathrm{e}+00$ & $5.680 \mathrm{e}+00$ & $5.677 \mathrm{e}+00$ & $1.062 \mathrm{e}+00$ & $8.037 \mathrm{e}+02$ & $9.496 \mathrm{e}+02$ \\
$F_{9}$ & $3.179 \mathrm{e}+00$ & $4.182 \mathrm{e}+00$ & $3.857 \mathrm{e}+00$ & $3.832 \mathrm{e}+00$ & $2.564 \mathrm{e}-01$ & $9.039 \mathrm{e}+02$ & $9.041 \mathrm{e}+02$ \\
$F_{10}$ & $2.662 \mathrm{e}+03$ & $2.215 \mathrm{e}+06$ & $1.837 \mathrm{e}+05$ & $3.348 \mathrm{e}+05$ & $5.022 \mathrm{e}+05$ & $2.680 \mathrm{e}+04$ & $8.762 \mathrm{e}+05$ \\
$F_{11}$ & $4.529 \mathrm{e}+00$ & $1.056 \mathrm{e}+01$ & $6.442 \mathrm{e}+00$ & $6.829 \mathrm{e}+00$ & $1.517 \mathrm{e}+00$ & $1.104 \mathrm{e}+03$ & $1.108 \mathrm{e}+03$ \\
$F_{12}$ & $4.781 \mathrm{e}+01$ & $3.759 \mathrm{e}+02$ & $2.058 \mathrm{e}+02$ & $1.883 \mathrm{e}+02$ & $9.524 \mathrm{e}+01$ & $1.235 \mathrm{e}+03$ & $1.429 \mathrm{e}+03$ \\
$F_{13}$ & $3.176 \mathrm{e}+02$ & $3.614 \mathrm{e}+02$ & $3.299 \mathrm{e}+02$ & $3.316 \mathrm{e}+02$ & $9.134 \mathrm{e}+00$ & $1.618 \mathrm{e}+03$ & $1.657 \mathrm{e}+03$ \\
$F_{14}$ & $1.880 \mathrm{e}+02$ & $2.151 \mathrm{e}+02$ & $2.035 \mathrm{e}+02$ & $2.033 \mathrm{e}+02$ & $6.743 \mathrm{e}+00$ & $1.592 \mathrm{e}+03$ & $1.603 \mathrm{e}+03$ \\
$F_{15}$ & $1.334 \mathrm{e}+01$ & $5.272 \mathrm{e}+02$ & $4.166 \mathrm{e}+02$ & $3.8762 \mathrm{e}+02$ & $1.611 \mathrm{e}+02$ & $1.514 \mathrm{e}+03$ & $1.900 \mathrm{e}+03$ \\
\hline
\end{tabular}

Performance eVAluation of C-EGPSO on $D=10$. CEC-2015 Benchmark FunCtions. Comparison with CMAES-R Results.

best results. Note that intuitively, there might be a link with the topology. Indeed, the topology used is the one proposed by Clerc in his technical report [7], where the number of neighbours is set to 3 . Thus, this is probably not a coincidence if using this topology where one third of the particles are best particles of the neighbourhood. However, we did not experiment for the case $4 / 4$ and more generally $\mathrm{n} / \mathrm{n}$ to confirm this intuition. How to select this coefficient in different optimisation problems will be further investigated in a future study.

\section{Experimental Results}

The obtained results are compiled in tables I and II. The best and mean results obtained by CMAES-R are also displayed for comparison. The best fitness of each function in the benchmark is computed following the procedure described in subection IV. All the selected problems are represented by their numbers in the CEC2015 benchmark. Figure 8 gives a graphical comparison with the results of other algorithms and will be studied through this subsection.

This investigation will deal with the ability of the proposed algorithm to perform on the presented problems in both of the studied dimensions $(D=10$ and $D=30$ ) and to compare with the results obtained from other algorithms. Figure 8 provides a compilation of the results obtained. They show the capacity of the proposed algorithms to converge to the solutions of the benchmark problems: C-EGPSO, CMAES-R, HumanCog, iSRPSO and MVMO.

Figure 8 demonstrates that for the two unimodal problems in the benchmark, the proposed algorithm did not find the global optimum within the limitation of 500 function calls. The ranges of values of these two functions are large and tend to flatten the solution area. Since there is no large difference between points in a same neighbourhood, the EGT cannot promote a specific search direction. It tends to equalize the three ESS coefficients and converge slowly. Nevertheless, the results should be scaled by the range of values. 


\begin{tabular}{|c|c|c|c|c|c|c|c|}
\hline Func & Best & Worst & Median & Mean & Std & CMAES-R Best & CMAES-R Mean \\
\hline$F_{1}$ & $2.005 \mathrm{e}+07$ & $3.032 \mathrm{e}+08$ & $1.554 \mathrm{e}+08$ & $1.514 \mathrm{e}+08$ & $9.342 \mathrm{e}+07$ & $9.691 \mathrm{e}+03$ & $1.289 \mathrm{e}+06$ \\
$F_{2}$ & $6.645 \mathrm{e}+04$ & $1.760 \mathrm{e}+05$ & $9.940 \mathrm{e}+04$ & $1.022 \mathrm{e}+05$ & $2.345 \mathrm{e}+04$ & $8.245 \mathrm{e}+04$ & $1.416 \mathrm{e}+05$ \\
$F_{3}$ & $2.095 \mathrm{e}+01$ & $3.301 \mathrm{e}+01$ & $2.773 \mathrm{e}+01$ & $2.746 \mathrm{e}+01$ & $3.739 \mathrm{e}+00$ & $3.185 \mathrm{e}+02$ & $3.253 \mathrm{e}+02$ \\
$F_{4}$ & $3.081 \mathrm{e}+03$ & $7.986 \mathrm{e}+03$ & $6.402 \mathrm{e}+03$ & $6.003 \mathrm{e}+03$ & $1.628 \mathrm{e}+03$ & $3.209 \mathrm{e}+03$ & $5.773 \mathrm{e}+03$ \\
$F_{5}$ & $2.030 \mathrm{e}+00$ & $4.705 \mathrm{e}+00$ & $3.480 \mathrm{e}+00$ & $3.376 \mathrm{e}+00$ & $6.778 \mathrm{e}-01$ & $5.005 \mathrm{e}+02$ & $5.041 \mathrm{e}+02$ \\
$F_{6}$ & $3.631 \mathrm{e}-01$ & $8.360 \mathrm{e}-01$ & $5.791 \mathrm{e}-01$ & $5.703 \mathrm{e}-01$ & $1.350 \mathrm{e}-01$ & $6.004 \mathrm{e}+02$ & $6.007 \mathrm{e}+02$ \\
$F_{7}$ & $2.303 \mathrm{e}-01$ & $1.101 \mathrm{e}+00$ & $3.706 \mathrm{e}-01$ & $4.883 \mathrm{e}-01$ & $2.640 \mathrm{e}-01$ & $7.003 \mathrm{e}+02$ & $7.006 \mathrm{e}+02$ \\
$F_{8}$ & $4.349 \mathrm{e}+01$ & $3.651 \mathrm{e}+03$ & $1.508 \mathrm{e}+02$ & $3.911 \mathrm{e}+02$ & $7.927 \mathrm{e}+02$ & $8.195 \mathrm{e}+02$ & $8.273 \mathrm{e}+02$ \\
$F_{9}$ & $1.259 \mathrm{e}+01$ & $1.384 \mathrm{e}+01$ & $1.334 \mathrm{e}+01$ & $1.331 \mathrm{e}+01$ & $3.686 \mathrm{e}-01$ & $9.135 \mathrm{e}+02$ & $9.139 \mathrm{e}+02$ \\
$F_{10}$ & $6.784 \mathrm{e}+05$ & $1.812 \mathrm{e}+07$ & $3.297 \mathrm{e}+06$ & $4.823 \mathrm{e}+06$ & $4.661 \mathrm{e}+06$ & $1.221 \mathrm{e}+06$ & $4.122 \mathrm{e}+06$ \\
$F_{11}$ & $2.069 \mathrm{e}+01$ & $1.114 \mathrm{e}+02$ & $2.437 \mathrm{e}+01$ & $3.514 \mathrm{e}+01$ & $2.610 \mathrm{e}+01$ & $1.119 \mathrm{e}+03$ & $1.136 \mathrm{e}+03$ \\
$F_{12}$ & $1.746 \mathrm{e}+02$ & $8.914 \mathrm{e}+02$ & $5.797 \mathrm{e}+02$ & $5.631 \mathrm{e}+02$ & $1.949 \mathrm{e}+02$ & $1.482 \mathrm{e}+03$ & $1.861 \mathrm{e}+03$ \\
$F_{13}$ & $3.715 \mathrm{e}+02$ & $4.714 \mathrm{e}+02$ & $4.117 \mathrm{e}+02$ & $4.151 \mathrm{e}+02$ & $2.382 \mathrm{e}+01$ & $1.671 \mathrm{e}+03$ & $1.693 \mathrm{e}+03$ \\
$F_{14}$ & $2.253 \mathrm{e}+02$ & $3.007 \mathrm{e}+02$ & $2.535 \mathrm{e}+02$ & $2.549 \mathrm{e}+02$ & $1.764 \mathrm{e}+01$ & $1.621 \mathrm{e}+03$ & $1.635 \mathrm{e}+03$ \\
$F_{15}$ & $8.513 \mathrm{e}+02$ & $1.178 \mathrm{e}+03$ & $9.887 \mathrm{e}+02$ & $1.014 \mathrm{e}+03$ & $8.678 \mathrm{e}+01$ & $1.940 \mathrm{e}+03$ & $2.296 \mathrm{e}+03$ \\
\hline
\end{tabular}

Performance eVAluation of C-EGPSO on $D=30$. CEC-2015 Benchmark FUnCtions. Comparison With CMAES-R Results.

Regarding the multimodal problems, C-EGPSO can obtain results comparable to the MVMO algorithm and outperforms CMAES-R on most of the selected problems. For functions with few and localised optima such like the HappyCat function of problem $F_{6}$, the principle to base the movement of the particles on the previous experience leads to the conclusion that the proposed approach enables the particles to "predict" this valley and the direction to follow to escape from a local optimum in order to converge to the global one. The problem $F_{8}$ features a large number of local optima. It allows the demonstration of the capacity of C-EGPSO to prevent a premature convergence by optimising the search direction. The particles are figuring out the shape of the solution space by basing their "assumptions" on their previous experiences and can shape a form of average mean of the solution space curvature, therefore avoiding to be trapped in local optima by selecting the best search direction to improve their fitness as shown in subection III-B. Moreover, basing the exploration on particle level instead of swarm level improves the reactivity of CEGPSO to converge compared to CMAES algorithms on multimodal functions.

The C-EGPSO efficiency on hybrid and composed problems can be explained by its average good performance on various types of multimodal functions and its ability to self-adapt to the solution space characteristics. However the adaptation of the three ESS coefficients is related to the complexity of the solution space. This could decrease the short-term performance of C-EGPSO for expensive problems.

It is noticeable that the consideration of Figure 8, which gives the results for $D=30$, shows that C-EGPSO keeps its quality on higher dimension even though the complexity is increasing, i.e. it is not sensitive to problem dimensionality.

Athough the MVMO algorithm obtains a better convergence on some of the problems of the CEC2015 benchmark, it needs to maintain an archive which is cost expensive whereas C-EGPSO only stores an updated history of its three coefficients. Moreover, as suggested in [17], the MVMO needs to tune numerous parameters to obtain interesting results. Therefore, the results presented in [17] were obtained with different parameters to optimised specifically for each problem while the same set of parameters was used by the C-EGPSO in this study.

\section{CONCLUSION}

The proposed algorithm introduces a new way to use evolutionary game theory in order to improve the particle swarm optimisation. The presented approach is based on the ability of the evolutionary game to optimise the search direction based on the previous experience of each particle while exploiting all the benefits from a classical particle swarm optimisation approach to avoid the local optima and explore the solution space. The algorithm has been tested on the benchmark functions found in the CEC-2015 congress. Its ability to have competitive performance on most of these problems has been demonstrated, especially the algorithm shows good adaptation skills. It can converge quickly to the optimum and can overcome local optima by adapting its ESS coefficients for each particle. In that extent, the C-EGPSO shows its capacity to improve the convergence speed and the ability to solve some deficiencies of SPSO 2011. This adaptibility also gives robust performance when the dimension increases or on hybrid problems. However, on problems featuring a plane topology, C-EGPSO converges more 
slowly, giving priority to space exploration. Taking into account the recent advances in parallel programming, this algorithm can be implemented in such a way that particles having not common neighbouring informants can perform their movements independently, hence the proposed algorithm is computationally parallelisable.

In the future, it might be interesting to explore deeper the evolutionary game element and look into the details how evolves the evolutionary stable strategy according to the initial state of the population. It could also be interesting to investigate the stability of these points. Since the selection of the criterion $p_{R}$, which denotes the probability for a particle to follow the classical SPSO 2011 or the proposed C-EGPSO, was empirically determined in this study, it could be interesting to investigate the role of this parameter in the global performance of the algorithm. The topology of the particles network could also be investigated by studying the Shapley's value in order to determine what is the role played by each particle in the entire swarm, then adapt the topology accordingly.

\section{REFERENCES}

[1] Congress on Evolutionary Computation, Sendai (JPN), May 2015.

[2] A. Al-Dujaili, K. Subramanian, and S. Suresh. Humancog: A cognitive architecture for solving optimization problems. In 2015 IEEE Congress on Evolutionary Computation (CEC), pages 3220-3227, May 2015.

[3] M. Andersson, S. Bandaru, A. Ng, and A. Syberfeldt. Parameter tuned cma-es on the cec'15 expensive problems. In Evolutionary Computation, 2015, pages 1950-1957, 2015.

[4] Q. Chen, B. Liu, Q. Zhang, J. J. Liang, P. N. Suganthan, and B. Y. Qu. Problem definitions and evaluation criteria for CEC 2015 special session on bound constrained single-objective computationally expensive numerical optimization. Technical report, Computational Intelligence Laboratory, Zhengzhou University, Zhengzhou, China and Technical Report, Nanyang Technological University, Singapore, Nov 2014, 2014.

[5] M. Clerc. Back to random topology. Technical report, http:// clerc.maurice.free.fr/pso/random_topology.pdf, 2007.

[6] M. Clerc. A method to improve standard PSO. Technical report, http://clerc.maurice.free.fr/pso/Design_efficient_PSO.pdf, 2009.

[7] M. Clerc. Standard particle swarm optimisation. Technical report, http://clerc.maurice.free.fr/pso/spso_descriptions.pdf, 2012.

[8] R. Cressman. Evolutionary Dynamics and Extensive Form Games. MIT Press, 2003.

[9] J. Kennedy and R. C. Eberhart. Particle swarm optimization. In Proceedings of IEEE International Conference on Neural Networks, pages 1942 - 1948, Piscataway (USA), 1995.

[10] J. Kennedy and R.C. Eberhart. A discrete binary version of the particle swarm algorithm. In The 1997 IEEE International Conference on Systems, Man, and Cybernetics, volume 5, pages 4104-4108, Orlando (USA), October 1997.

[11] C. Leboucher, R. Chelouah, P. Siarry, and S. Le Ménec. A swarm intelligence method combined to evolutionary game theory applied to the resources allocation problem. International Journal of Swarm Intelligence Research, 3(2):20 - 38, 2012.

[12] C. Leboucher, H-S. Shin, P. Siarry, R. Chelouah, S. Le Menec, and A. Tsourdos. A two-step optimisation method for dynamic weapon target assignment problem, recent advances on metaheuristics and their application to real scenarios. In Dr. Javier Del Ser Lorente, editor, recent advances on metaheuristics and their application to real scenarios, chapter 10, pages 266-290. InTech, 2013.
[13] C. Leboucher, H-S Shin, P. Siarry, S. Le Ménec, R. Chelouah, and A. Tsourdos. Convergence proof of an enhanced particle swarm optimisation method integrated with evolutionary game theory. Information Sciences, 346:389-411, 2016.

[14] J. Maynard-Smith. Evolution and the theory of games. Cambridge University Press, 1982.

[15] R. Mendes. Population topologies and their influence in particle swarm performance. $\mathrm{PhD}$ thesis, University Minho, 2004.

[16] K. C. Monson and K. C. Seppi. Exposing origin -seeking bias in PSO. In GECCO'05, pages 241 - 248, Washington (USA), 2005.

[17] J. L. Rueda and I. Erlich. Testing mvmo on learning-based realparameter single objective benchmark optimization problems. In 2015 IEEE Congress on Evolutionary Computation (CEC), pages 1025-1032, May 2015.

[18] W. Sandholm. Population Games and Evolutionary Dynamics. MIT Press, 2010.

[19] M. R. Tanweer, S. Suresh, and N. Sundararajan. Improved srpso algorithm for solving cec 2015 computationally expensive numerical optimization problems. In 2015 IEEE Congress on Evolutionary Computation (CEC), pages 1943-1949, May 2015.

[20] P. Taylor and Jonker L. Evolutionary stable strategies and game dynamics. Mathematical Bioscience, 40:145-156, 1978. 
2018-01-03

\title{
An enhanced particle swarm optimization method integrated with evolutionary game theory
}

\author{
Leboucher, Cédric
}

IEEE

Cédric Leboucher, Hyo-Sang Shin, Rachid Chelouah, et al., An enhanced particle swarm optimization method integrated with evolutionary game theory. IEEE Transactions on Games, 2018 Volume 10, Issue 2, pp. 221-230 https:doi.org/10.1109/TG.2017.2787343

Downloaded from Cranfield Library Services E-Repository 